not be favourable for observations of disturbances so far $\left(80^{\circ} \mathrm{W}\right.$.) off the central meridian.

University of Western Australia,

S. E. WILLIAMS

$$
\text { Nedlands, W.A. }
$$

1 Martyn, Nature, 159, 26 (1947).

${ }^{2}$ Shklovsky, Nature, 159, 752 (1947).

3 Appleton and Hey, Phil. Mag., 37, 73 (1946).

Allen, private communication.

6 Wtllams and Hands, Nature, 158, 511 (1946).

\section{Photo-electric Fourier Transformer and its Application to Sound-Films}

AT the time of the publication of the accounts by Born, Fürth and Pringle ${ }^{1,2}$ of the new instrument named by them the 'photo-electric Fourier transformer', we happened to be engaged in constructing a very similar instrument here. The principle of the apparatus and the main essentials of its design had been worked out by one of us (D. B.) in continuation of earlier work on acoustic spectra from sound-films ; it seemed probable that the method would have advantages over the diffraction method previously employed ${ }^{3}$.

Between our arrangement and that of the above authors the only significant differences are as follow. (a) We do not employ masks or silhouettes representing the functions to be analysed, as it has always been our aim to use actual sound-film. (b) Instead of producing the Fourier transforms of given functions singly, we seek to produce a continuous or running analysis as one function changes by gradual transitions into another. Our apparatus, therefore, includes a provision for moving the record of the function $A$ (that is, the sound-film) steadily past the optical aperture, and simultaneously moving the photographic film $B$ upon which the transformed function is recorded or transcribed. (c) The signals from the photocell are employed to give intensity modulation on the oscillograph screen instead of amplitude modulation; this is necessitated by (b) above. (d) In view of the fact that the higher audio-frequencies are registered on a sound-film as extremely fine periodicities indeed, we employ a grating with a greater number of more closely spaced elements; our grating is in the form of a disk 6 in. in diameter with 55 elements to the inch.

In other respects, such as the general arrangement of the disk, slit, photocell and oscillograph, and the use of a harmonic time-base synchronized electromagnetically with the rotating disk, our method coincides surprisingly closely with that of the above authors.

Early attempts to produce a satisfactory disk were made during the war period, but the work had then to be abandoned for more urgent matters. Shortly after the project was resumed, the paper of Born, Fürth and Pringle appeared, but we continued our work as we had a somewhat different object in view in constructing the instrument. Incidentally, when this is to be used to analyse actual sound-film, the requirements of the optical and amplifier systems become very stringent, presenting problems which could not be overcome immediately. The accompanying photographs show, however, that it is possible, using this apparatus, to display sound in the desired way. When a film record of speech sound is subjected to analysis, a succession of frequency-versustime patterns is obtained, of the type illustrated by Fig. 1, which represents the word 'there', containing the diphthong $\varepsilon+$ ə. From previous knowledge,
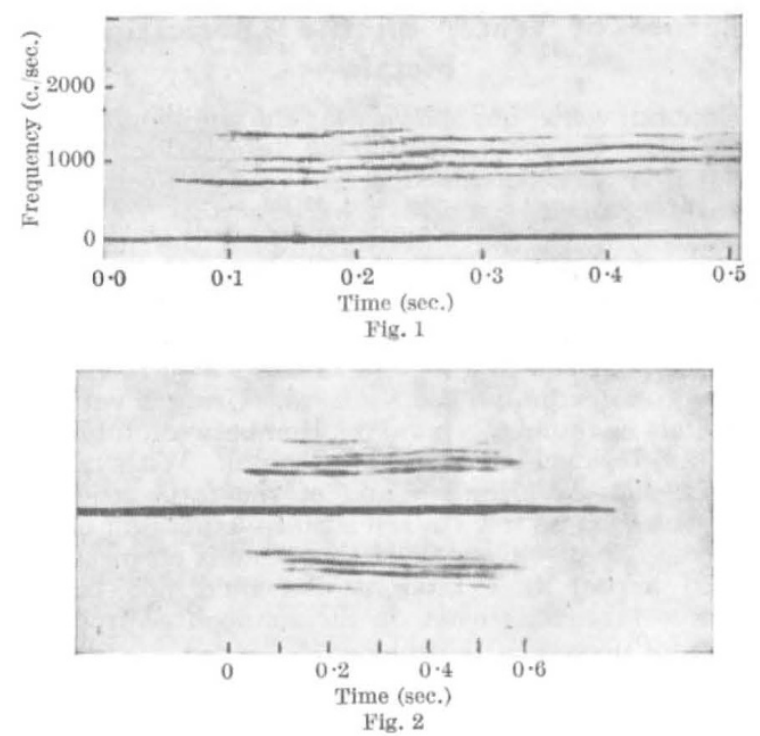

the acoustic spectrum would be expected to show a considerable number of harmonics of the vocal-cord pitch, with certain of the harmonics augmented in intensity by the resonances of the vocal cavities, and we find these characteristics clearly brought out in the photograph. Some pre-distortion in favour of the higher frequencies was employed during recording, and for this reason, and because of its low natural intensity, the fundamental pitch itself is not registered in this trace.

The zero-frequency line in the record is produced by the steady component of the light transmitted. by the sound film. Neglecting small phase-difference effects, the oscillograph pattern is symmetrical about this line, so that if the whole pattern is photographed, the effect is as shown in Fig. 2, which is derived from the same initial film record as Fig. 1. Also, Fig. 2 illustrates the effect of using a smaller velocity for the film $B$ in transcribing, so that the time-scale is more compressed. The changes in the vocal resonance regions, also the swing of the harmonic frequencies away from the axis, due to rising pitch inflexion, are now more easily appreciated by the eye.

It is not proposed to discuss here possible applications of this method of presenting vibration phenomena, which, of course, need not be limited to acoustic vibrations. In the field of communications, reference may be made to the recent survey by Gabor ${ }^{4}$. Also, while the present work was in progress, a series of articles has appeared ${ }^{5}$ describing some remarkable recent developments in the Bell Telephone Laboratories, and their applications. The soundspectrograph there described achieves in a striking manner the result aimed at in our own investigations, a fuller account of which will be published elsewhere. In conclusion, we wish to acknowledge great assistance from Mr. C. F. Coleman in the conduct of these experiments.

D. Brown

J. W. LXTTLETON

Auckland University College,

Auckland, C.1, Now Zealand. July 31 .

${ }^{1}$ Born, M., Fürth R., and Pringle, R. W., Nature, 156, 756 (1945).

2 Fürth, R., and Pringle, R. W., Phil. Mag., 37, 1 (1946).

'Brown, D., Proc. Phys. Soc. Lond., 51, 244 (1939).

- Gahor, D., J. Inst. Elect. Eng., 93, 429 (1946).

'J. Acoust. Soc. Amer., 18, 1 (1946). 